\title{
\begin{tabular}{l|l} 
Mitraries & DSpace@MIT
\end{tabular}
}

\author{
MIT Open Access Articles
}

\section{Feedback schemes for radiation damping suppression in NMR: a control-theoretical perspective}

The MIT Faculty has made this article openly available. Please share how this access benefits you. Your story matters.

Citation: Altafini, C., P. Cappellaro, and D. Cory. “Feedback schemes for radiation damping suppression in NMR: a control-theoretical perspective." Decision and Control, 2009 held jointly with the 2009 28th Chinese Control Conference. CDC/CCC 2009. Proceedings of the 48th IEEE Conference on. 2009. 1445-1450. (C) 2009 IEEE

As Published: http://dx.doi.org/10.1109/CDC.2009.5400761

Publisher: Institute of Electrical and Electronics Engineers

Persistent URL: http://hdl.handle.net/1721.1/58824

Version: Final published version: final published article, as it appeared in a journal, conference proceedings, or other formally published context

Terms of Use: Article is made available in accordance with the publisher's policy and may be subject to US copyright law. Please refer to the publisher's site for terms of use. 


\title{
Feedback schemes for radiation damping suppression in NMR: a control-theoretical perspective
}

\author{
C. Altafini, P. Cappellaro, and D. Cory
}

\begin{abstract}
In NMR spectroscopy, the collective measurement is weakly invasive and its back-action is called radiation damping. The aim of this paper is to provide a controltheoretical analysis of the problem of suppressing this radiation damping. It is shown that the two feedback schemes commonly used in the NMR practice correspond one to a high gain oputput feedback for the simple case of maintaining the spin $1 / 2$ in its inverted state, and the second to a 2-degree of freedom control design with a prefeedback that exactly cancels the radiation damping field. A general high gain feedback stabilization design not requiring the knowledge of the radiation damping time constant is also investigated.
\end{abstract}

\section{INTRODUCTION}

In recent years, the theory [23], [12], [15], [19], [21] and practice [17] of (real-time) feedback for quantum mechanical systems has gained momentum especially in contexts such as quantum optics [23]. In order to avoid wavefunction collapse, the measurement is assumed weak and the feedback is seen as a way to influence the resulting dynamics conditioned by the measurement back-action. This conditioning is stochastic for a single isolated quantum system [21], but can assume the form of a deterministic back-action when considering the expectation values for an ensemble of systems [19], [20], [13]. In this last setting, the effect of a weak measurement is described by a term in a Markovian master equation which can be conservative (when the measurement is perfect, i.e., lossless) or dissipative (imperfect measurement).

In NMR spectroscopy, in presence of a collective spin measurement the phenomenon occurring is called radiation damping [8], [9], [6], and it is due to the electromagnetic field induced by the current passing through the detection coil while doing a measurement. This field in turn interacts with the spins in the sample, hence it induces a back-action on the system observed. If back-actions are hallmarks of quantum measurement, magnetic resonance is no exception in this respect.

In high field and probes of high quality factor, radiation damping is typically an important effect only at certain frequency ranges, for example that of the abundant spin of the solvent. For these, it behaves much like a soft pulse, steering the magnetization vector back to its equilibrium value. For other bandwidths, the back-action signal is so weak it is dominated by the relaxation effects, and hence it

C. Altafini (corresponding author) is with SISSA-ISAS International School for Advanced Studies, via Beirut 2-4, 34014 Trieste, Italy, altafiniesissa.it

P. Cappellaro is with ITAMP, Harvard University, 60 Garden Street, MS 14 Cambridge, MA 02138, pcappel laro@cfa.harvard.edu

D. Cory is with Dept. of Nuclear Eng., MIT, 150 Albany St., Cambridge, MA 02139-4307, dcory@mit.edu is negligible. In this work we assume to be dealing with one of those situations in which radiation damping is of interest and relaxation is negligible.

A model of radiation damping exists since the fifties [8], [9], and assumes that the back-action is conservative, i.e., it preserves the norm of the Bloch vector. Efforts to engineer the NMR receiving/transmitting system in order to reject this form of back-action have been going on for more than a decade and by now there are many ways to compensate for it, such as electronic feedback [10], [16], [2], [14], rf pulse compensation [11], gradient field, Q-switches, and composite pulse sequences, see [6], [14] for a more detailed survey. We are here interested only in the first two methods.

The aim of this paper is threefold. First, we provide a rigorous convergence analysis of the behavior induced by the radiation damping effect and described qualitatively in several papers [1], [3], [6], [7], [18]. Second, we aim to give a system-theoretic interpretation of the electronic feedback and pulse compensation control designs. We will show that the first scheme suppresses the radiation damping field by canceling the current in the coil. In its so-called "École Polytechnique design", [14], this can be thought of as an exact feedback matching problem, i.e., a precompensator based on an internal model of the radiation damping field. While this requires the exact knowledge of the radiation damping time constant, a high gain variant of the same problem can be set up in order to maintain the spin $1 / 2$ in the "fully inverted" state, although it works only for this particular state. For generic states, the exact cancellation of the radiation damping dynamics alone does not achieve asymptotic stabilization. However, it can be intended as a prefeedback to which a second active field can be linearly superimposed, in order to produce desired control actions. In control terms, this design is called a 2-degrees of freedom (DOF) control design, and resembles the schemes described in [11], [14].

The third and last aim of this paper is to explore possible alternative/improved schemes inspired by control theory. In the spirit of feedback control, we show that the 2-DOF design mentioned above can be completed with an extra feedback loop, allowing to achieve closed-loop asymptotic stabilization, a more robust concept than just exact canceling by matching. We will further see that also a high gain state feedback can be designed in order to achieve tracking of a desired trajectory up to a limited steady state tracking error. Unlike the 2 DOF scheme based on exact radiation damping cancellation, this last feedback controller does not require the explicit knowledge of the radiation damping time 
constant. For "high gain" we mean a ratio of around an order of magnitude between the actuation current and the current produced by the spin precession. Hence the task of radiation damping compensation can be performed in the soft pulse regime, meaning that real-time feedback makes sense in this context even with a single coil available. When strong pulses are instead considered, the above transmitter/receiver ratio is several orders of magnitude higher, hence alternative designs such as, for example, an interleaved scheme of pulsing and measuring, should be used instead.

\section{THE MODEL FOR RADIATION DAMPING}

In the following, we shall consider the model of radiation damping described e.g. in [8], [9], [6], [3], [22], focusing only on the spin $1 / 2$ case. Further details concerning the model formulation are available in the Appendix.

Disregarding relaxation effects (i.e., in the limit $T_{1}=$ $\left.T_{2}=\infty\right)$ and denoting with $\mathbf{m}=\left[m_{x} m_{y} m_{z}\right]^{T}$ the normalized Bloch vector, $\left(\mathbf{m}=\mathbf{M} / M_{o}\right.$ where $M_{o}$ is the equilibrium magnetization), the nonlinear Bloch equations for radiation damping in a frame rotating with the circuit resonant frequency are

$$
\begin{aligned}
\frac{d m_{x}}{d t} & =\delta m_{y}-\ell m_{x} m_{z} \\
\frac{d m_{y}}{d t} & =-\delta m_{x}-\ell m_{y} m_{z} \\
\frac{d m_{z}}{d t} & =\ell\left(m_{x}^{2}+m_{y}^{2}\right)
\end{aligned}
$$

where $\delta=\omega-\omega_{o}$ is the offset between the Larmor precession frequency $\omega_{0}$ and the circuit resonant frequency $\omega, \ell$ is the radiation damping rate $\ell=\frac{1}{T_{R}}$, with $T_{R}$ the radiation damping time constant $T_{R}=\frac{\gamma}{2 \pi \xi M_{o} Q}(\gamma=$ gyromagnetic ratio, $\xi=$ coil filling factor, $Q=$ probe quality factor) [9], [6], [3]. Denoting $A_{x}, A_{y}$ and $A_{z}$ the real rotation matrices around the $x, y$, and $z$ axis, $\operatorname{Lie}\left(A_{x}, A_{y}, A_{z}\right)=$ $\operatorname{span}\left(A_{x}, A_{y}, A_{z}\right)=\mathfrak{s o}(3)$, then (1) can be written as

$$
\frac{d \mathbf{m}}{d t}=-\delta A_{z} \mathbf{m}+\ell\left\langle\left\langle\mathbf{m}_{o}, A_{x} \mathbf{m}\right\rangle\right\rangle A_{x} \mathbf{m}+\ell\left\langle\left\langle\mathbf{m}_{o}, A_{y} \mathbf{m}\right\rangle\right\rangle A_{y} \mathbf{m}
$$

where $\mathbf{m}_{o}=\left[\begin{array}{ll}0 & 0\end{array}\right]^{T}$ is the north pole of the Bloch sphere (aligned with the static magnetic field applied to the ensemble) and $\langle\langle\cdot, \cdot\rangle\rangle$ denotes an Euclidean inner product in $\mathbb{R}^{3}$.

Proposition 1: The system (2) has $\mathbf{m}_{o}$ as an almost globally asymptotically stable equilibrium point, with region of attraction $\mathbb{S}^{2} \backslash\left\{\mathbf{m}_{1}\right\}$, where $\mathbf{m}_{1}=\left[\begin{array}{ll}0 & -1\end{array}\right]^{T}$ is the inverted state.

Proof: Consider the $\mathbb{S}^{2}$-distance

$$
V=\|\mathbf{m}\|-\left\langle\left\langle\mathbf{m}_{o}, \mathbf{m}\right\rangle\right\rangle .
$$

Clearly $V(\mathbf{m})>0 \forall \mathbf{m} \in \mathbb{S}^{2} \backslash\left\{\mathbf{m}_{o}\right\}, V\left(\mathbf{m}_{o}\right)=0$. Differentiating along the trajectories of (2):

$$
\begin{aligned}
\dot{V}= & -\left\langle\left\langle\mathbf{m}_{o}, \dot{\mathbf{m}}\right\rangle\right\rangle \\
= & \delta\left\langle\left\langle\mathbf{m}_{o}, A_{z} \mathbf{m}\right\rangle\right\rangle-\ell\left\langle\left\langle\mathbf{m}_{o}, A_{x} \mathbf{m}\right\rangle\right\rangle\left\langle\left\langle\mathbf{m}_{o}, A_{x} \mathbf{m}\right\rangle\right\rangle \\
& -\ell\left\langle\left\langle\mathbf{m}_{o}, A_{y} \mathbf{m}\right\rangle\right\rangle\left\langle\left\langle\mathbf{m}_{o}, A_{y} \mathbf{m}\right\rangle\right\rangle .
\end{aligned}
$$

Since $A_{z} \mathbf{m}_{o}=\left[\begin{array}{lll}0 & 0 & 0\end{array}\right]^{T}$, the first term disappears and hence

$$
\dot{V}=-\ell\left\langle\left\langle\mathbf{m}_{o}, A_{x} \mathbf{m}\right\rangle\right\rangle^{2}-\ell\left\langle\left\langle\mathbf{m}_{o}, A_{y} \mathbf{m}\right\rangle\right\rangle^{2} \leqslant 0 .
$$

Therefore $V(\cdot)$ is a Lyapunov function for the equilibrium $\mathbf{m}_{o}$ of (2). As $\dot{V}=0$ only for $\mathbf{m}=\mathbf{m}_{o}$ or $\mathbf{m}=\mathbf{m}_{1}, \mathbf{m}_{o}$ is an attractor for (2) with basin of attraction $\mathbb{S}^{2} \backslash\left\{\mathbf{m}_{1}\right\}$.

It is straightforward to check that the inverted state $\mathbf{m}_{1}$ is an unstable equilibrium of (2). In fact, in the literature, it is known that a weak perturbation or even a noise disturbing $\mathbf{m}$ can trigger the coherent radiation from $\mathbf{m}_{1}$ to the lower energy state $\mathbf{m}_{o}[18],[7]$.

\section{Feedback COnTRol StRategies}

For a coil aligned for instance with the laboratory $x$ axis, the measured NMR signal is a current which is generated by the electromotive force (emf) induced in the coil by the precessing magnetization $\mathbf{m}$ and which oscillates with the spin resonance frequency $\omega_{o}$. This may be superimposed with another emf due to the external driving, i.e., to the control input (soft pulses regime only). These two oscillating emfs (or, in the AC steady state, the two corresponding oscillating currents, see Appendix for details) give rise to two magnetic fields. In the rotating frame, denote with $\phi$ the field due to the spin precession and with $\mathbf{u}$ the externally driven field, respectively of components $\phi_{x}, \phi_{y}$ and $u_{x}, u_{y}$. From (2), we have

$$
\left\{\begin{array}{l}
\phi_{x}(\mathbf{m})=\ell\left\langle\left\langle\mathbf{m}_{o}, A_{x} \mathbf{m}\right\rangle\right\rangle=\ell m_{y} \\
\phi_{y}(\mathbf{m})=\ell\left\langle\left\langle\mathbf{m}_{o}, A_{y} \mathbf{m}\right\rangle\right\rangle=-\ell m_{x} .
\end{array}\right.
$$

Including $\mathbf{u}$ in the model (2), we have

$\frac{d \mathbf{m}}{d t}=-\delta A_{z} \mathbf{m}+\left(u_{x}+\phi_{x}(\mathbf{m})\right) A_{x} \mathbf{m}+\left(u_{y}+\phi_{y}(\mathbf{m})\right) A_{y} \mathbf{m}$.

The following proposition is obvious at least in one direction.

Proposition 2: For the system (5), the norm of the transverse magnetization is constant $\forall t$ if and only if $u_{i}=$ $-\phi_{i}(\mathbf{m}), i=x, y$.

Proof: The condition $u_{i}=-\phi_{i}(\mathbf{m}), i=x, y$ implies that $\dot{\mathbf{m}}=-\delta A_{z} \mathbf{m}$ which leaves the transverse magnetization invariant. For the other direction, $m_{x}^{2}+m_{y}^{2}=1-m_{z}^{2}=$ const $\forall t$ implies that $\dot{m}_{z}=0$. From (1) and (2), this yields

$$
\begin{aligned}
& \ell\left(m_{x}^{2}+m_{y}^{2}\right)+u_{x} m_{y}-u_{y} m_{x}=0 \\
& \quad \forall m_{x}, m_{y} \text { such that } m_{x}^{2}+m_{y}^{2}=\text { const }
\end{aligned}
$$

i.e.,

$$
\begin{aligned}
& \left(\ell m_{x}-u_{y}\right) m_{x}+\left(\ell m_{y}+u_{x}\right) m_{y}=0 \\
& \forall m_{x}, m_{y} \text { such that } m_{x}^{2}+m_{y}^{2}=\text { const. }
\end{aligned}
$$

This is satisfied only when $\ell m_{x}-u_{y}=0$ and $\ell m_{y}+u_{x}=0$ simultaneously.

The electronic feedback suppression of radiation damping of [10] (denoted "Brussels scheme" in [14], Fig. 1, middle) works on the current induced by the spin precession in the coil ( $I_{\text {spin }}$ in the notation of the Appendix) and suppresses 


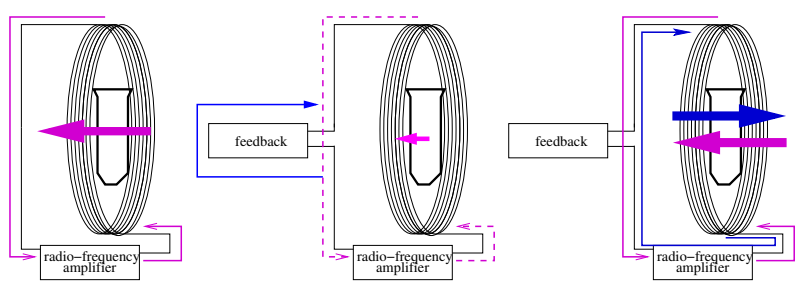

Fig. 1. Cartoons of the electronic feedback compensation schemes for radiation dampings. Left: non-compensated radiation damping. The arrow represents the back-action field acting on the spin ensemble. Middle: "Brussels scheme". The radiation damping current is suppressed in the coil. Right: "École Polytechnique scheme". The current is compensated for in the coil.

it through a suitable circuit, see [14]. From (11), suppressing this current (or reducing it by 2-3 orders of magnitude) means suppressing the corresponding field $\phi$ and hence the radiation damping backaction, see (5). This is done in a completely electronic manner, not requiring the use of $\mathbf{u}$ and it is said in [14] that in the residual (small) current the signalto-noise ratio is essentially unaltered.

An alternative scheme, called "École Polytechnique scheme" in [14] (Fig. 1, right), works by detecting, inverting and suppressing $\phi$ through the rf generator and hence through $\mathbf{u}$, see [16], [2] for the original papers. In our formalism, this corresponds to the feedback of Proposition 2. The need of fine-tuning of the rf current $I_{r f}$ for this scheme corresponds to the fact that the exact cancellation of Proposition 2 requires the knowledge of $\ell$ (and hence of the radiation damping time constant $T_{R}$ ).

A particular subtask, valid only for the inverted state $\mathbf{m}_{1}$, can however be carried out without the explicit knowledge of $\ell$ by means of a high gain feedback law.

Proposition 3: For the system (5), the feedback

$$
\begin{aligned}
& u_{x}=-k \phi_{x}(\mathbf{m}) \\
& u_{y}=-k \phi_{y}(\mathbf{m})
\end{aligned}
$$

$k>1$, renders the inverted state $\mathbf{m}_{1}$ almost globally asymptotically stable.

Proof: Consider the Lyapunov function of (3) with respect to $\mathbf{m}_{1}, V=\|\mathbf{m}\|-\left\langle\left\langle\mathbf{m}_{1}, \mathbf{m}\right\rangle\right\rangle$, and differentiate it

$$
\begin{aligned}
\dot{V}= & -\left\langle\left\langle\mathbf{m}_{1}, \dot{\mathbf{m}}\right\rangle\right\rangle \\
= & -\left(u_{x}+\phi_{x}(\mathbf{m})\right)\left\langle\left\langle\mathbf{m}_{1}, A_{x} \mathbf{m}\right\rangle\right\rangle \\
& -\left(u_{y}+\phi_{y}(\mathbf{m})\right)\left\langle\left\langle\mathbf{m}_{1}, A_{y} \mathbf{m}\right\rangle\right\rangle \\
= & -(1-k) \phi_{x}(\mathbf{m})\left\langle\left\langle\mathbf{m}_{1}, A_{x} \mathbf{m}\right\rangle\right\rangle \\
& -(1-k) \phi_{y}(\mathbf{m})\left\langle\left\langle\mathbf{m}_{1}, A_{y} \mathbf{m}\right\rangle\right\rangle \\
= & (1-k)\left\langle\left\langle\mathbf{m}_{1}, A_{x} \mathbf{m}\right\rangle\right\rangle^{2}+(1-k)\left\langle\left\langle\mathbf{m}_{1}, A_{y} \mathbf{m}\right\rangle\right\rangle^{2} \leqslant 0
\end{aligned}
$$

since e.g. $\phi_{x}(\mathbf{m})=\left\langle\left\langle\mathbf{m}_{o}, A_{x} \mathbf{m}\right\rangle\right\rangle=-\left\langle\left\langle\mathbf{m}_{1}, A_{x} \mathbf{m}\right\rangle\right\rangle$.

This use of the "École Polytechnique scheme" for the feedback stabilization of the inverted state is also mentioned in [14]. Observe that when $u_{x}=u_{y}=0$, one has $\dot{V}>0$ i.e., the equilibrium $\mathbf{m}_{1}$ indeed becomes unstable, as already mentioned after Proposition 1.

\section{A. 2-DOF with feedback stabilization}

From the proof of Proposition 3, if $k=1$ then the evolution is only stable but not an attractor $(\dot{V}=0$ in absence of external controls) and, from Proposition 2, this corresponds to exact cancellation of the radiation damping. More generally, we may be interested in manipulating the spin state while suppressing at all times the effect of radiation damping. In the "Brussels scheme", this can be carried out by simply setting $\phi=0$ in (5), and building a suitable feedback law for $\mathbf{u}$. In the "École Polytechnique scheme", we can adopt a 2-degrees of freedom (DOF) control design composed of a prefeedback that cancels the unwanted dynamics linearly superimposed with a controller that achieves the desired task, e.g. stabilize the state to the desired orbit of the drift term (i.e., a horizontal circle characterized by a desired value of $m_{z}$ ). The general scheme for such a 2-DOF control design is given by

$$
\left\{\begin{array}{l}
u_{x}=-\phi_{x}(\mathbf{m})+v_{x} \\
u_{y}=-\phi_{y}(\mathbf{m})+v_{y}
\end{array}\right.
$$

with $v_{x}, v_{y}$ the new control variables. This 2 DOF controller is the one proposed in [11] (similar arguments also appear in [14]). The feedback design of $v_{x}, v_{y}$ can for example follow the theory developed in [4]. As in [4], we shall not try to suppress the precession motion (which would introduce singularities in the control law). Rather, we will formulate the stabilization to the orbit given by the desired value of $m_{z}$, call it $m_{d, z}$, as a state tracking problem for the dynamical trajectory described by the following system

$$
\frac{d \mathbf{m}_{d}}{d t}=-\delta A_{z} \mathbf{m}_{d}
$$

The following proposition formalizes this result: a trajectory stabilizing state feedback superimposed with the prefeedback of Proposition 2 achieves asymptotic stabilization of $\mathbf{m}$ to $\mathbf{m}_{d}$.

Proposition 4: Consider the system (5). The 2 DOF feedback controller given by (7) and

$$
\left\{\begin{array}{l}
v_{x}=k\left\langle\left\langle\mathbf{m}_{d}, A_{x} \mathbf{m}\right\rangle\right\rangle \\
v_{y}=k\left\langle\left\langle\mathbf{m}_{d}, A_{y} \mathbf{m}\right\rangle\right\rangle
\end{array}\right.
$$

$k>0$, tracks the reference trajectory $\mathbf{m}_{d}$ given by (8) in an asymptotically stable manner for all $\mathbf{m}(0) \in \mathbb{S}^{2}$ with the exception of the antipodal point $\mathbf{m}(0)=-\mathbf{m}_{d}(0)$ and of $\mathbf{m}(0), \mathbf{m}_{d}(0)$ both lying on great horizontal circles.

Proof: Consider the candidate Lyapunov function

$$
V=\left\|\mathbf{m}_{d}\right\|^{2}-\left\langle\left\langle\mathbf{m}_{d}, \mathbf{m}\right\rangle\right\rangle
$$

and differentiate it:

$$
\begin{aligned}
\dot{V}= & -\left\langle\left\langle\dot{\mathbf{m}}_{d}, \mathbf{m}\right\rangle\right\rangle-\left\langle\left\langle\mathbf{m}_{d}, \dot{\mathbf{m}}\right\rangle\right\rangle \\
= & \delta\left\langle\left\langle A_{z} \mathbf{m}_{d}, \mathbf{m}\right\rangle\right\rangle+\delta\left\langle\left\langle\mathbf{m}_{d}, A_{z} \mathbf{m}\right\rangle\right\rangle \\
& \quad-v_{x}\left\langle\left\langle\mathbf{m}_{d}, A_{x} \mathbf{m}\right\rangle\right\rangle-v_{y}\left\langle\left\langle\mathbf{m}_{d}, A_{y} \mathbf{m}\right\rangle\right\rangle \\
= & -k\left(\left\langle\left\langle\mathbf{m}_{d}, A_{x} \mathbf{m}\right\rangle\right\rangle^{2}+\left\langle\left\langle\mathbf{m}_{d}, A_{y} \mathbf{m}\right\rangle\right\rangle^{2}\right) \leqslant 0
\end{aligned}
$$

where the cancellation of the two drift terms occurs since $A_{z}^{T}=-A_{z}$. Hence the reference trajectory $\mathbf{m}_{d}(t)$ is at least 
stable. The proof of convergence and the analysis of the basin of attraction is now formally identical to that carried out in Proposition 1 of [4] (see also examples in [5]).

\section{B. Compensating without knowledge of $\ell$}

The feedback controller in Proposition 4 requires: i) full state information (i.e., the on-line knowledge of the Bloch vector, retrievable by numerical integration of (5)); ii) the knowledge of $\ell$ (i.e., of the time constant $T_{R}$ of the radiation damping). The interesting question is whether a high gain feedback scheme (similar to Proposition 3) can be obtained without the explicit knowledge of $\ell$ for the more general task studied in Proposition 4.

Proposition 5: Consider the system (5) and the reference trajectory (8). Assuming that the radiation damping rate $\ell$ is unknown, the system with the state feedback

$$
\left\{\begin{array}{l}
u_{x}=k\left\langle\left\langle\mathbf{m}_{d}, A_{x} \mathbf{m}\right\rangle\right\rangle \\
u_{y}=k\left\langle\left\langle\mathbf{m}_{d}, A_{y} \mathbf{m}\right\rangle\right\rangle
\end{array}\right.
$$

$k>0$, converges to an orbit which approaches the reference trajectory (8) when $k$ is large. The steady state tracking error (i.e., the $\mathbb{S}^{2}$-distance between the two orbits) is $1-$ $\left(k+\ell m_{d, z}\right) / \sqrt{k^{2}+\ell^{2}+2 k \ell m_{d, z}}$.

Proof: Once again, the argument is based on a Lyapunov function, but for a reference trajectory $\mathbf{M}_{f}=k \mathbf{m}_{d}+\ell \mathbf{m}_{o}$. From (8) $\frac{d \mathbf{M}_{f}}{d t}=-k \delta A_{z} \mathbf{m}_{d}$, but, since $A_{z} \mathbf{m}_{o}=0$, also $\frac{d \mathbf{M}_{f}}{d t}=-k \delta A_{z} \mathbf{M}_{f}$. Since, tipically, $\mathbf{M}_{f} \notin \mathbb{S}^{2}$, consider $\mathbf{m}_{f}=\frac{\mathbf{M}_{f}}{\left\|\mathbf{M}_{f}\right\|}$, where $\left\|\mathbf{M}_{f}\right\|=\sqrt{k^{2} m_{d, x}^{2}+k^{2} m_{d, y}^{2}+\left(k m_{d, z}+\ell\right)^{2}}=$ $\sqrt{k^{2}+\ell^{2}+2 k \ell m_{d, z}}$, and, consequently, $\frac{d \mathbf{m}_{f}}{d t}=-\delta A_{z} \mathbf{m}_{f}$. This expression implies that considering $V_{f}=\|\mathbf{m}\|^{2}-$ $\left\langle\left\langle\mathbf{m}_{f}, \mathbf{m}\right\rangle\right\rangle$ and differentiating, the drift terms disappear and we have

$$
\begin{aligned}
\dot{V}_{f} & =-\left\langle\left\langle\dot{\mathbf{m}}_{f}, \mathbf{m}\right\rangle\right\rangle-\left\langle\left\langle\mathbf{m}_{f}, \dot{\mathbf{m}}\right\rangle\right\rangle \\
& -\left\langle\left\langle\mathbf{m}_{f},\left(u_{x}+\phi_{x}\right) A_{x} \mathbf{m}\right\rangle\right\rangle-\left\langle\left\langle\mathbf{m}_{f},\left(u_{y}+\phi_{y}\right) A_{y} \mathbf{m}\right\rangle\right\rangle \\
& =-\left\langle\left\langle\mathbf{m}_{f}, A_{x} \mathbf{m}\right\rangle\right\rangle^{2}-\left\langle\left\langle\mathbf{m}_{f}, A_{y} \mathbf{m}\right\rangle\right\rangle^{2} \leqslant 0
\end{aligned}
$$

i.e., we have convergence to $\mathbf{m}_{f}=\left(k \mathbf{m}_{d}+\ell \mathbf{m}_{o}\right) /\left\|\mathbf{M}_{f}\right\|$. As $\mathbf{m}_{d}(t)$ is symmetrically distant from $\mathbf{m}_{o} \forall t$, also $\mathbf{m}_{f}(t)$ is so, meaning that to compute the distance between the attractor orbit $\mathbf{m}_{f}$ and the desired one $\mathbf{m}_{d}$ a simple $\mathbb{S}^{2}$ distance can be used, regardless of the initial condition:

$$
\begin{aligned}
d\left(\mathbf{m}_{f}, \mathbf{m}_{d}\right) & =1-\left\langle\left\langle\mathbf{m}_{f}, \mathbf{m}_{d}\right\rangle\right\rangle \\
& =1-\left\langle\left\langle k \mathbf{m}_{d}+\ell \mathbf{m}_{o}, \mathbf{m}_{d}\right\rangle\right\rangle /\left\|\mathbf{M}_{f}\right\| \\
& =1-\left(k+\ell\left\langle\left\langle\mathbf{m}_{o}, \mathbf{m}_{d}\right\rangle\right\rangle\right) /\left\|\mathbf{M}_{f}\right\| \\
& =1-\left(k+\ell m_{d, z}\right) / \sqrt{k^{2}+\ell^{2}+2 k \ell m_{d, z}} .
\end{aligned}
$$

It is clear from Proposition 5 that when the feedback gain $k$ is high (say an order of magnitude higher than $\ell$ ), the steady state tracking error becomes negligible, in particular near the equator. In Fig. 2 we show an example of how this steady state tracking error shrinks when the gain $k$ is

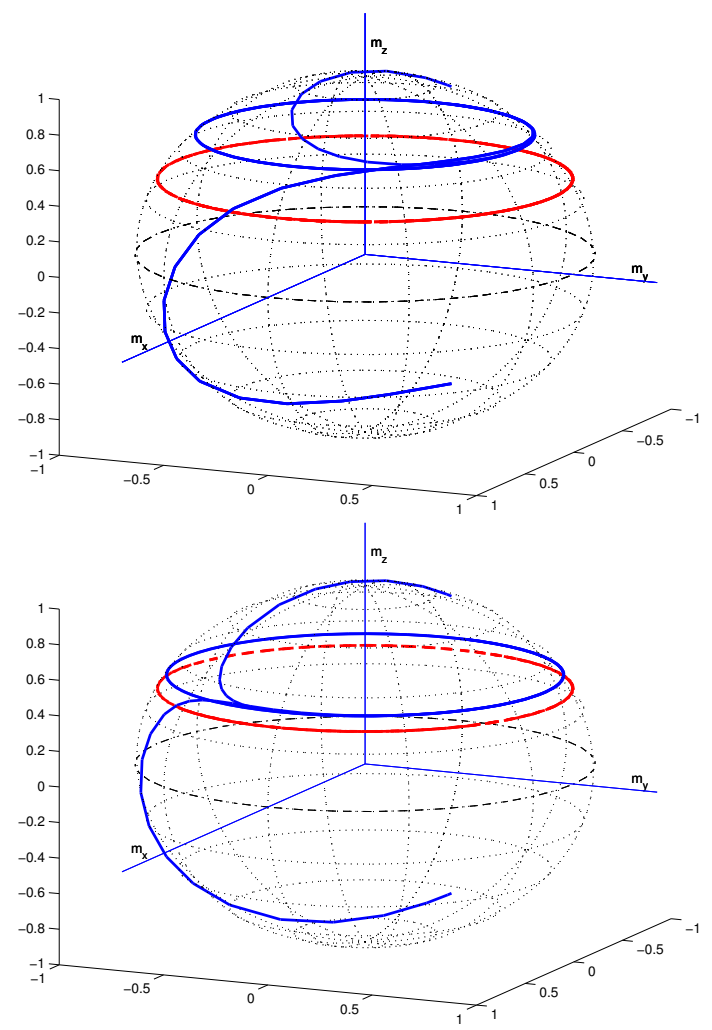

Fig. 2. High gain state feedback stabilization without radiation damping exact compensation. The two plots show each two curves of the system (5) with the feedback (10) from different initial conditions (color on-line: blue solid lines). Clearly both converge to a orbit that is different from the desired one of (8) (color on-line: red dashed line). However, in the right plot where a higher gain is used this orbit is closer to the desired one than on the left plot (the ratio of the two gains is 4 ; the higher value of $k$ is 10 times $\ell$ ).

increased. Notice how this tracking error depends on the sign of $m_{d, z}$ and is larger for orbits on the lower hemisphere, see Fig. 3.

\section{CONCLUSION}

As for many other aspects of the NMR literature, we find that also the methods developed for the purpose of suppressing radiation damping admit nontrivial control theoretical formulations. Part of the aim of this paper is to translate this problem and its solutions into language and techniques familiar to a control audience. In particular, we obtain that feedback control strategies can be classified into two types of methods: high gain feedback and 2 DOF controllers with a prefeedback exactly canceling the radiation damping term. We also show how to use the first type of controller for more general tasks than considered in the literature, while still not requiring exact knowledge of the time constant of radiation damping (a necessary condition for the methods based on exact cancellation). As for the 2 DOF control design, we show how this can be completed to a true feedback stabilizer that achieves a desired task in an asymptotically stable manner. 

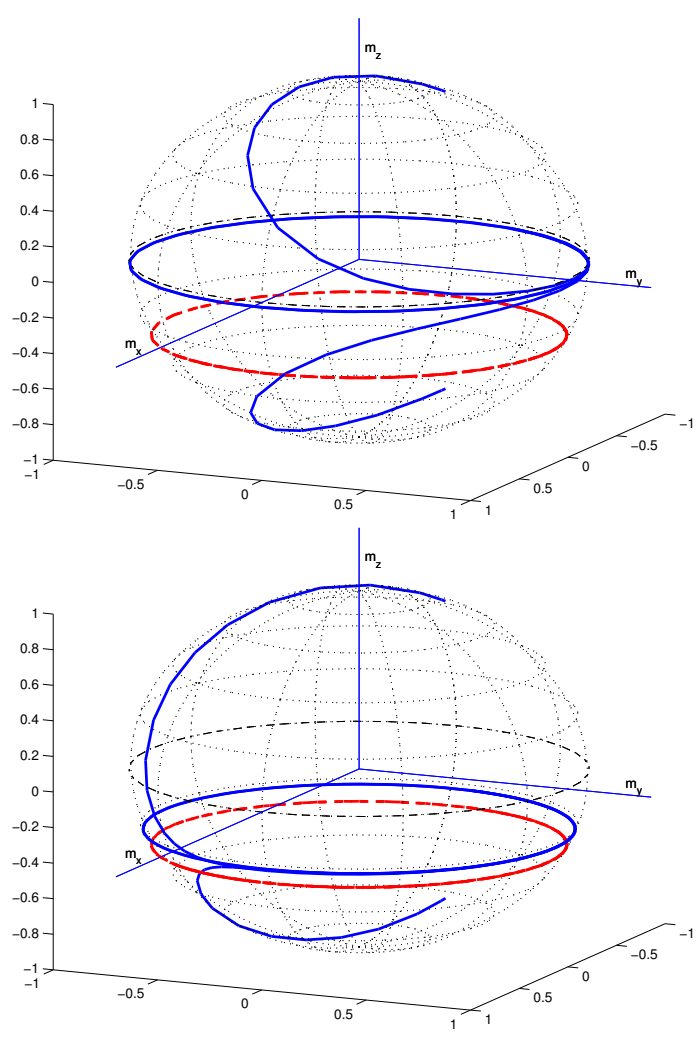

Fig. 3. Steady state tracking error depends on the sign of $m_{d, z}$. The two figures show the same tracking problem as in Fig. 2, with the only difference that now $m_{d, z}$ has negative sign. Proposition 5 predicts that the steady state tracking error is larger than in the case of Fig. 2. This is particularly visible for the low gain situation (left plot).

\section{APPENDIX}

In this Section we follow essentially [6]. The collective effect of the ensemble of spins precessing around the $z$ axis is to induce an electromotive force in the receiver coil. In the laboratory frame, according to Faraday's law, this oscillating voltage can be expressed as

$$
V_{\text {spin }}(t)=-4 \pi \xi \eta A \frac{d \mathfrak{M}_{x}(t)}{d t}
$$

where $\eta$ is the number of turns in the coil, $\xi$ is the filling factor, $A$ is the cross-sectional area of the coil, and $\mathfrak{M}_{x}$ is the component of the magnetization vector aligned with the laboratory $x$-axis (conventionally the axis of the coil). Applying Kirchhoff's law to this coil

$$
L \frac{d^{2} I(t)}{d t^{2}}+R \frac{d I(t)}{d t}+\frac{I(t)}{C}=\frac{d}{d t}\left(V_{\text {spin }}+V_{r f}\right)
$$

where $V_{r f}$ is the external voltage applied by the generator (i.e., the control input). The natural frequency of the circuit is $\omega=\frac{1}{\sqrt{L C}}$ and its quality factor $Q=\omega L / R$. In the low $Q$ limit, we can assume (following e.g. [22]), that the damping part of this damped harmonic oscillator is quickly exhausted, and work at the resulting AC steady state, assuming that also the external signal $V_{r f}$ is a soft pulse (i.e., slowly varying in the rotating frame). This leads to $V_{\text {spin }}+V_{r f}=Z I(t)$, where the impedance $Z$ is a function of $\omega, \omega_{o}, Q$, and $L$ [22]. Special devices, such as the directional coupler circuit described in [14], allow to distinguish in $I(t)$ the two components due to the spin precession and to the external generator: $I(t)=I_{\text {spin }}(t)+I_{r f}(t)$. Each oscillating current induces a field. In the frame rotating with the circuit frequency $\omega$, the two fields are denoted $\mathbf{u}$ and $\phi$. For example,

$$
I_{\text {spin }}(t)=\sqrt{\frac{\mathrm{Vol}}{\pi L}} \phi \cos (\omega t+\psi)
$$

where Vol is the coil volume and $\psi$ is the phase of the current. As the components of $\mathbf{m}$ are related to $\mathfrak{M}_{x}$ by

$$
\mathfrak{M}_{x}=m_{x} \cos (\omega t)+m_{y} \sin (\omega t),
$$

the components of the field $\phi$ correspond to the expressions in (4).

\section{REFERENCES}

[1] D. Abergel. Chaotic solutions of the feedback driven bloch equations. Phys. Lett. A., 302:17-22, 2002.

[2] D. Abergel, C. Carlotti, A. Louis-Joseph, and J.-Y. Lallemand. Improvements in radiation-damping control in high-resolution nmr. Journal of Magnetic Resonance, Series B, 109(2):218 - 222, 1995.

[3] D. Abergel, A. Louis-Joseph, and J. Lallemand. Self-sustained Maser oscillations of a large magnetization driven by a radiation dampingbased electronic feedback. J. Chem. Phys., 116(16):7073-7080, 2002.

[4] C. Altafini. Feedback control of spin systems. Quantum Information Processing, 6:9-36, 2007.

[5] C. Altafini. Feedback stabilization of isospectral control systems on complex flag manifolds: application to quantum ensembles. IEEE Transactions on Automatic Control, 52(11):2019-2028, 2007.

[6] M. Augustine. Transient properties of radiation damping. Progr. Nucl. Magn. Res. Spectr., 40(2):111-150, 2002.

[7] M. Augustine, S. Bush, and E. Hahn. Noise triggering of radiation damping from the inverted state. Chem. Phys. Lett., 322(1-2):111-118, 2000.

[8] N. Bloembergen and R. V. Pound. Radiation damping in magnetic resonance experiments. Physical Review, 95(1):8-12, 1954.

[9] S. Bloom. Effects of radiation damping on spin dynamics. J. Applied Physics, 28(7):800-805, 1957.

[10] P. Broekaert and J. Jeener. Suppression of radiation damping in NMR in liquids by active electronic feedback. J. Magn. Res. A, 113(1):6064, MAR 1995.

[11] J. Chen, B. Cutting, and G. Bodenhausen. Measurement of radiation damping rate constants in nuclear magnetic resonance by inversion recovery and automated compensation of selective pulses. J. Chem. Phys., 112(15):6511-6514, APR 152000.

[12] A. Doherty, K. Jacobs, and G. Jungman. Information, disturbance and hamiltonian quantum feedback control. Phys. Rev. A, 63:062306, 2001.

[13] N. Ganesan and T. J. Tarn. Decoherence control in open quantum systems via classical feedback. Phys. Rev. A, 75:032323, 2007.

[14] J. Jeener. Collective effects in liquid NMR: Dipolar field and radiation damping. Encyc. Nucl. Magn. Res., 9:642-679, 2002.

[15] S. Lloyd and J. Slotine. Quantum feedback with weak measurements. Phys. Rev. A, 62(1), 2000.

[16] A. Louis-Joseph, D. Abergel, and J.-Y. Lallemand. Neutralization of radiation damping by selective feedback on a $400 \mathrm{mhz} \mathrm{nmr}$ spectrometer. Journal of Biomolecular NMR, 99, 1995.

[17] H. Mabuchi. Coherent-feedback quantum control with a dynamic compensator. Physical Review A, 78:032323, 2008.

[18] A. Sodickson, W. E. Maas, and D. G. Cory. The Initiation of Radiation Damping by Noise. J. Magnetic Resonance, B, 110:298-303, 1996.

[19] L. Thomsen, S. Mancini, and H. Wiseman. Continuous quantum nondemolition feedback and unconditional atomic spin squeezing. $J$. Phys. B-At. Mol. Opt. Phys., 35(23):4937-4952, 2002.

[20] F. Ticozzi and L. Viola. Quantum markovian subsystems: invariance, attractivity and control. IEEE Tr. Autom. Contr., 53:2048-2063, 2008 
[21] R. van Handel, J. K. Stockton, and H. Mabuchi. Feedback control of quantum state reduction. IEEE8 Trans. Automat. Control, 50:768-780, 2005.

[22] A. Vlassenbroek, J. Jeener, and P. Broekaert. Radiation damping in high resolution liquid NMR: A simulation study. The Journal of Chemical Physics, 103(14):5886-5897, 1995.

[23] H. M. Wiseman and G. J. Milburn. Quantum theory of optical feedback via homodyne detection. Phys. Rev. Lett., 70:548-551, 1993. 